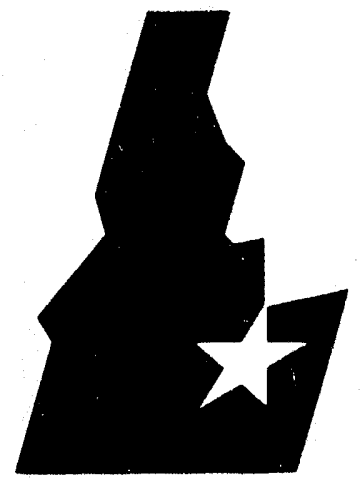

Idaho

National

Engineering

Laboratory

Managed

by the U.S.

Department

of Energy
EGG-ER-10792

June 1993

Rovision 0

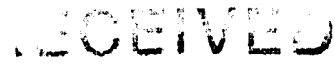

NOV 15153

OSTI

\title{
ACCEPTANCE SAMPLING METHODS FOR SAMPLE RESULTS \\ VERIFICATION
}

Carole A. Jesse

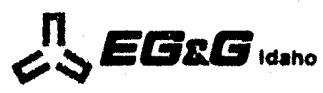

Work performed under DOE Contract No. DE-ACO7-761D01570 
EGG-ER-10792

Revision 0

\title{
Acceptance Sampling Methods
}

\section{for SAMPLe Results Verification}

\author{
Carole A. Jesse
}

Published June 1993

\section{Idaho National Engineering Laboratory EG\&G IDAHO, INC. IDAHO FALLS, ID 83415}

Prepared for the

U.S. Department of Energy

Assistant Secretary for Environmental Restoration and Waste Management Under DOE Idaho Operations Office Contract DE-ACO7-761D01570 


\section{Acceptance Sampling Methods}

\section{for Sample Results Verification}

June 1993

Prepared by

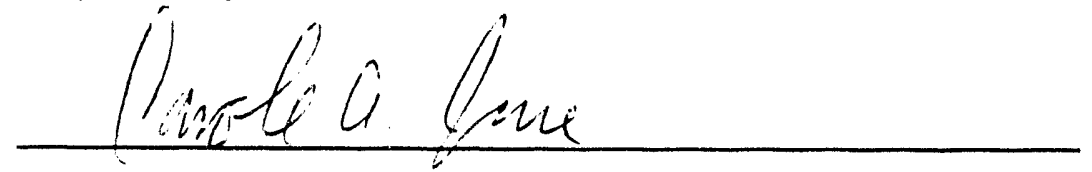

C. A. Jesse, Author

Statistics, Reliability, and Analys is Unit

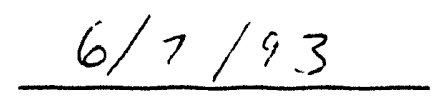

Date

Reviewed by
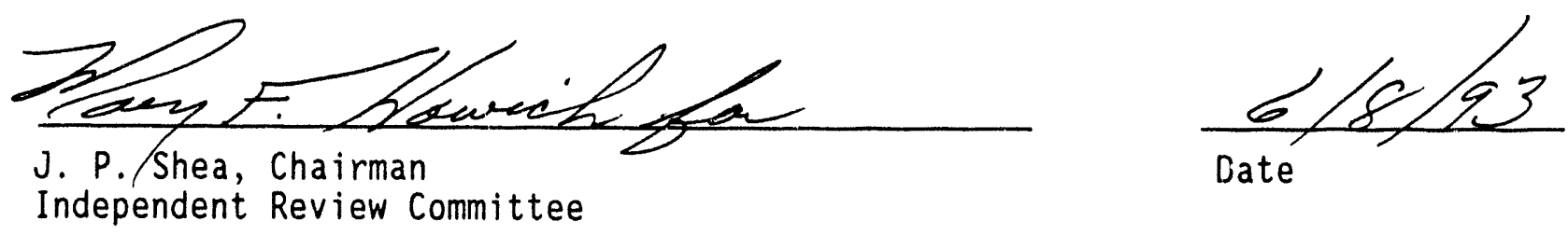

Date

Independent Review Committee

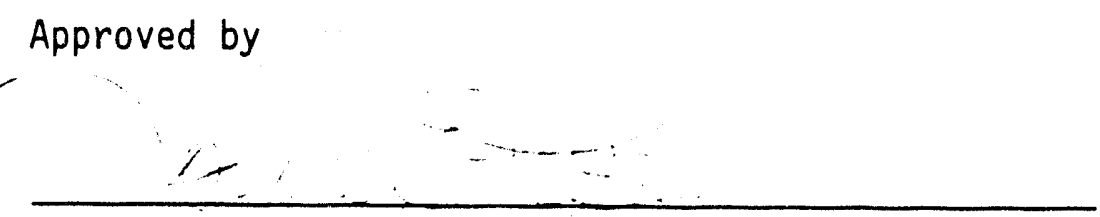

J. E. Férguson, Manager

Information Systems and Laboratory Management Unit

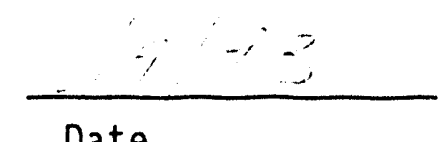

\section{DISCLAIMER}

This report was prepared as an account of work sponsored by an agency of the United States Government. Neither the United States Government nor any agency thereof, nor any of their employees, makes any warranty, express or implied, or assumes any legal liability or responsibility for the accuracy, completeness, or usefulness of any information, apparatus, product, or process disclosed, or represents that its use would not infringe privately owned rights. Reference herein to any specific commercial product, process, or service by trade name, trademark, manufacturer, or otherwise does not necessarily constitute or imply its endorsement, recominendation, or favoring by the United States Government or any agency thereof. The views and opinions of authors expressed herein do not recessarily state or reflect those of the United States Government or any agency thereof. 


\begin{abstract}
This report proposes a statistical sampling method for use during the sample results verification portion of the validation of data packages. In particular, this method was derived specifically for the validation of data packages for metals target analyte analysis performed under United States Environmental Protection Agency Contract Laboratory Program protocols, where sample results verification can be quite time consuming. The purpose of such a statistical method is to provide options in addition to the "all or nothing" options that currently exist for sample results verification. The proposed method allows the amount of data validated during the sample results verification process to be based on a balance between risks and the cost of inspection.
\end{abstract}




\section{ACKNOWLEDGEMENTS}

The author wishes to acknowledge the help of Mark Bryan, whose computer programming skills, participation in discussions of the statistical methodology, and editorial comments/suggestions were invaluable to the generation of this report. The author also wishes to thank Robert Sheehan for acting as a generous source of information on the data validation process, and providing key input necessary to this work. 


\section{CONTENTS}

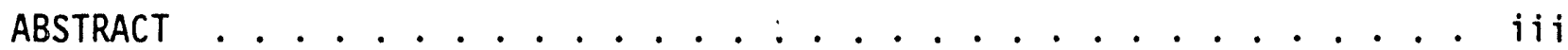

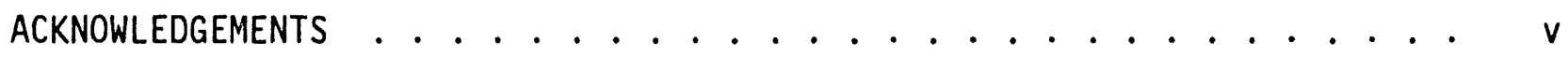

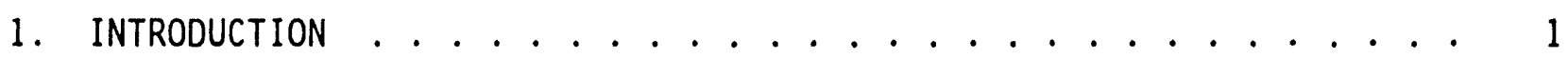

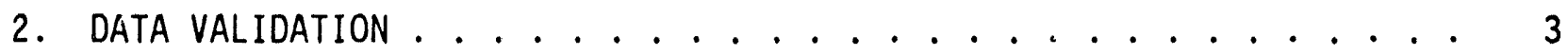

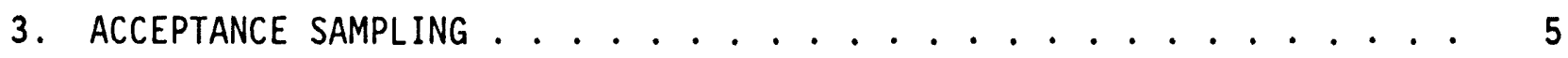

3.1 General ...................... 5

3.2 Consumer's and Producer's Risks ........... 6

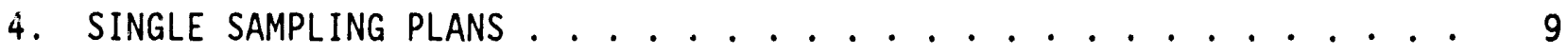

4.1 Introduction . . . . . . . . . . . . . . . 9

4.2 The Single Sampling Plans for Sample Results Verification . . 9

4.3 The Assumptions Associated With the Single Sampling Plans . . 11

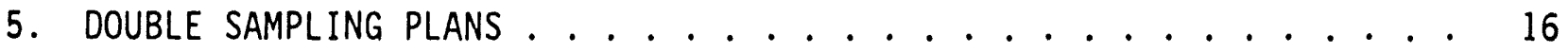

6. RECOMMENDATIONS ............................. 17

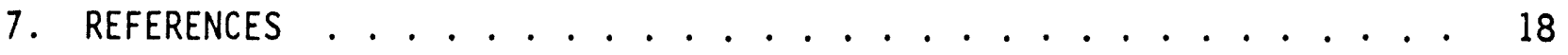

APPENDIX A SINGLE SAMPLING PLAN TABLES ............. A-1

APPENDIX B MATHEMATICAL STATISTICS OF THE SINGLE SAMPLING PLANS . . . B B-1

APPENDIX C MATHEMATICAL STATISTICS OF THE DOUBLE SAMPLING PLANS . . . C c-1 


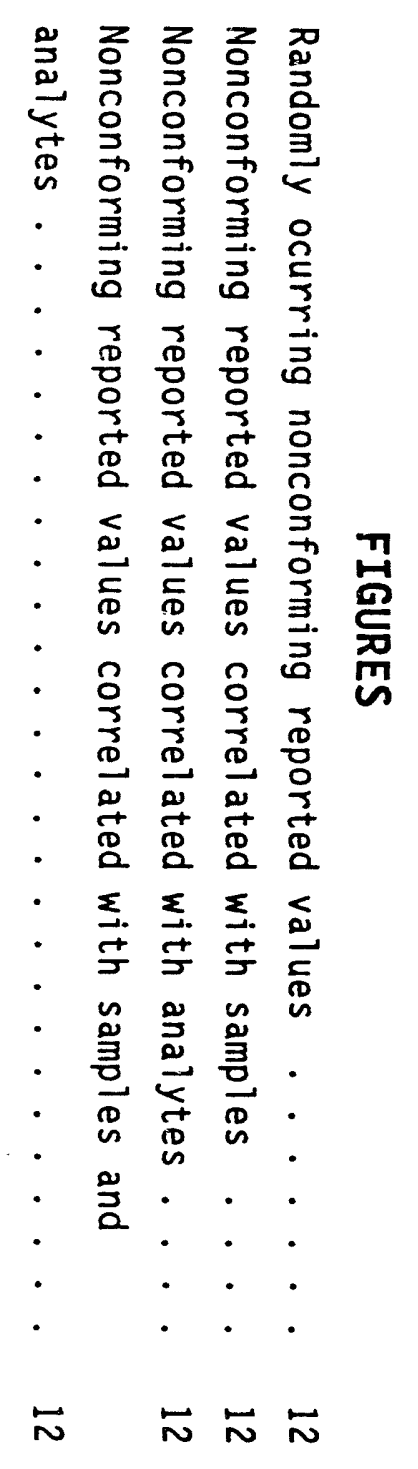




\section{Acceptance Sampling Methods for Sample Results Verification}

\section{INTRODUCTION}

This report describes a statistical sampling method for implementation in the Sample Results Verification (SRV) portion of data validation which is performed by the Sample Management office (SMO). In particular, this method was derived specifically for the validation of data packages for metals target analyte analysis performed under United States Environmental Protection Agency Contract Laboratory Program (CLP) protocols, where SRV can be quite time consuming. The purpose of such a statistical method is to provide options in addition to the "all or nothing" options that currently exist for SRV. The proposed method allows the amount of data validated during the SRV process to be based on a balance between risks and the cost of inspection.

The report begins with a discussion of the data validation process. Then Section 3 introduces the concept of acceptance sampling and illustrates how it applies to SRV. The specifics of the acceptance sampling plans are covered in Sections 4 and 5, and Appendix A. A discussion of recommendations can be found in Section 6 . The technical details of the mathematical statistics are left to Appendix $B$ and $C$, for those readers who may be interested.

To avoid massive confusion between the two distinct uses of the word sample in this report, the following convention will be used throughout:

Sample:

The use of the word sample will be the same as that employed by the SMO. In other words, sample will be used when referring to a portion of material to be analyzed that is contained in a single or multiple containers and identified by a unique sample number. 


\section{Statistical Sample:}

The term statistical sample will be used when referring to a subset of distinct units which have been drawn from a population. For this report statistical sample will always refer to a subset of the reported values contained in a data package. 


\section{DATA VALIDATION}

Data validation is the process of evaluating the quality and reliability of data produced during laboratory analyses. The SMO is responsible for the validation of data packages that are generated under CLP protocols.

The management of data, from the identification of initial need to the data delivery, occurs in the following way. A project manager determines that existing data for a given operable unit is inadequate to answer their questions regarding risk to human health or the environment. A Sampling and Analysis Plan is then developed which specifies how many samples are to be collected, and how they are to be gathered and analyzed for particular analytes in question. The finite, predetermined number of samples required for the project are collected over a given time period from a particular site. The term case refers to the entire collection of samples needed for the project.

As the samples from one case are submitted to the contract laboratory they are grouped into sample delivery groups. A sample delivery group (SDG) consists of 20 or fewer samples of the same matrix (i.e. soil or water) from one case sent to the contract laboratory, over a 14 day period, for the same analysis. A case will consist of one or more SDGs. The data package which is generated on an SDG by the contract laboratory will include the analysis results of all samples in the SDG including field samples, dilutions, reanalyses, blanks, spikes, duplicates, and laboratory control samples.

The data package associated with an SDG includes, for each sample, a form (Form 1) containing the analyte concentration values from the analysis of the sample. An analyte concentration value contained on a Form 1 is here after referred to as a reported value. For the remainder of this report, the term sample delivery group (SDG), when used, will refer specifically to the entire collection of reported values contained in a data package.

The extent of data validation required for a case is usually determined by the project manager, based on the intended use of the data. Currently the four 
data validation levels are $A, B, C$, and $X$, as defined in SMO-SOP-12.1.1. Essentially the data validation level determines the extent of validation to be done on the data packages comprising the project manager's case. There are many parts to the complicated process of data validation and they are dictated by the data validation level.

The objective of the SRV portion of data validation is to ensure that the reported values accurately represent those values generated in the laboratory for a particular sample. In the case of inorganic analyses on soil/sediment matrix samples, there is extensive transformation of the raw data in order to arrive at the reported values on the Form 1's. SRV consists of verifying this transformation process for an individual reported value as well as comparing the reported value to the calibration range and instrument detection limit. SRV can be a substantial part of data validation when the samples are of a soil or sediment matrix submitted for inorganic analysis.

For a Level A data validation, which is the most stringent validation level, SRV is required on all reported values in the data package. Data validation levels $B, C$, and $X$, do not require any SRV, and in fact parts of the SRV are impossible to perform for data validation level $X$ due to the unavailability of raw data. The main purpose of this report is to provide the project manager and/or the validator with other options beyond the "all" (level A) or "nothing" (levels B and C) requirements that currently exist for SRV. The statistical method used to develop these other options is based on acceptance sampling as discussed in the next section. 


\section{ACCEPTANCE SAMPLING}

\subsection{General}

Acceptance Sampling is a quality control method for determining the acceptability of a batch of manufactured product after it has been produced. In this context the term batch refers to a collection of manufactured product made within a specified time frame under like conditions. The producer of the manufactured product (the Producer) must assure the buyer (the Consumer) that the batch meets or exceeds the consumer's requirements. An individual unit of product (unit) which does not meet the consumer's requirements is considered nonconforming, otherwise it is considered to be a conforming unit. One measure of the quality of a batch is the fraction of nonconforming units, expressed as a percentage (here after referred to as the fraction nonconforming and denoted by $p$ ). Since it is very difficult to eliminate all process variation, the fraction nonconforming will vary from one batch to the next. The consumer wants the fraction nonconforming to be controlled but is usually willing to assign a maximum allowable fraction nonconforming that they are willing to tolerate.

One-hundred percent inspection with rectification is an inspection procedure where all the units in the batch are inspected and nonconforming units are replaced with conforming ones, thus guaranteeing the fraction nonconforming to be zero. In many instances one-hundred percent inspection is impractical since the cost of inspecting the product can be high and/or the inspection process may actually destroy the product (e.g. tensile testing). When these circumstances exist, only a subset (statistical sample) of the batch is inspected. The results from inspecting the statistical sample are then used to infer the nature of the entire batch. Acceptance sampling methods encompass this type of inspection procedure where statistical sampling of the batch is employed.

In the application of acceptance sampling to data validation, a single SDG represents a batch and the unit of product to be inspected is an individual reported value. The Producer is the laboratory generating the SDGs, and the Consumer is the project manager or the ultimate user of the reported values. 
During SRV a reported value is checked for the nonconformities that are listed in a standard operating procedure (SMO-SOP-12.1.5, Section $X$ ). When a reported value exhibits one or more of these nonconformities it is considered nonconforming. Attempts are made to correct the problem if possible, otherwise the reported value may be qualified by the validator.

One-hundred percent inspection is the requirement for SRV on a level A validation. To move from one-hundred percent inspection to an acceptance sampling approach to SRV it is necessary to understand the risks involved. The risks associated with acceptance sampling methods are discussed in the next section.

\subsection{Consumer's and Producer's Risks}

One-hundred percent inspection does not involve statistical sampling and therefore has no potential for either falsely accepting or falsely rejecting a batch. The previous statement assumes the inspection process itself is perfect (conforming units are always deemed good, and nonconforming units are always deemed bad), and this is also an underlying assumption used throughout this report. When the outcome of inspecting a statistical sample is used to infer the nature of an entire batch the potential for erroneous decisions increases, therefore the risks of false acceptance and false rejection become non-zero.

False acceptance of an SDG means that it has been deemed acceptable when in fact the proportion of nonconforming reported values is worse than the maximum allowable that the project manager will tolerate. The likelihood, or probability of false acceptance associated with an acceptance sampling plan is called the Consumer's Risk. Similarly, false rejection of an SDG means that it has been deemed unacceptable and has been rejected, when in fact the proportion of nonconforming reported values is no worse than the maximum allowable defined by the project manager. The probability of false rejection associated with an acceptance sampling plan is called the Producer's Risk. Both Consumer's and 
Producer's Risks are expressed as percentages in this report. An incremental decrease in either the Consumer's or Producer's Risk typically requires an increase in the size of the statistical sample or an increase in the other risk.

In terms of SRV, the project manager may be willing to tolerate a maximum allowable fraction nonconforming, denoted by $p_{1}$, in the SDGs of a case. This maximum allowable fraction nonconforming should be dictated by the criticality of the data and its end use, similar to the data validation level. If an SDG is rejected based on a statistical sample, the resulting action taken by the validator is to inspect all the reported values in the SDG. By doing so the validator will know if false rejection has taken place. If false rejectior has occurred it means the SDG was rejected when in fact the fraction nonconforming is no worse than $p_{1}$. The consequence of false rejection is the unnecessary cost incurred by the project manager from the resulting one-hundred percent inspection. Thus, the application of acceptance sampling to data validation is unusual in that the project manager, the Consumer, is directly affected by the Producer's Risk. The project manager will want an acceptance sampling plan with a small Producer's Risk. It is worth noting that while small Producer's Risk helps avoid unnecessary one-hundred percent inspection it also requires a larger statistical sample size.

Contrary to the case of false rejection, the validator will not know if false acceptance has occurred. Careful thought should be given to the ramifications of false acceptance of an SDG, i.e., what are the consequences of accepting an SDG with a fraction nonconforming worse than $p_{1}$ ? The consequences of false acceptance include the potential for incorrect decisions, based on the reported values, regarding the risks to human health and the environment caused by the contaminant levels present at a site. These consequences can be great. The project manager will want an acceptance sampling plan where the likelihood of false acceptance is low (small Consumer's Risk) for the chosen $p_{1}$.

There are many different types of acceptance sampling plans. The type of acceptance sampling plan alluded to in the previous discussion, one statistical sample drawn from the batch for inference purposes, is called a single sampling 
plan. Other types of acceptance sampling plans may involve two (double sampling) or more (multiple sampling) statistical samples. This report deals only with the development of single and double sampling plans for the SRV application. 


\section{SINGLE SAMPLING PLANS}

\subsection{INTRODUCTION}

To determine the acceptability of a batch using a single sampling plan for attributes, a statistical sample is randomly drawn from the batch and inspected. If the number of nonconforming units in the statistical sample, denoted by $x$, exceeds a predetermined number, the batch is rejected, otherwise it is accepted. The number of units in the batch is derioted by $N$ and the number of units inspected (statistical sample size) is denoted by $n$. The predetermined number, corresponding to the maximum number of nonconforming units that are acceptable in the statistical sample, is denoted by $A C$ and is called the acceptance criterion. Since rejection of the batch occurs if the number of nonconforming units in the statistical sample is $A c+1$ or more, the rejection criterion is denoted ly $R e=A c+1$. A single sampling plan is completely specified by $n$ and Ac.

\subsection{The Single Sampling Plans for Sample Results Verification}

Appendix A contains tables of single sampling plans for the validation of SDGs through the SRV process. Each table is associated with a specific value of the maximum allowable fraction nonconforming, denoted by $p_{1}, t_{i}$ be chosen by the project manager. Tables 1,2 , and 3 are based on a $p_{1}$ of $5 \%, 10 \%$, and $20 \%$, respectively.

Each table in Appendix A has six columns. The first column gives the SDG size $(N)$. An individual sample delivery group submitted for metals target analyte analysis will contain between 1 and 20 samples, each analyzed for 23 analytes (24 if cyanide is included, which is rare). As a result, the number of reported values to be validated in a SDG runs between 23 and 460 by increments of 23 , i.e., $N=23,46,69, \ldots 460$. The second and third columns provide the statistical sample size $(n)$ and acceptance criterion $(A c)$, respectively, of the single sampling plan corresponding to the SDG size. 
The calculations of the Consumer's and Producer's Risks (fourth and fifth column, respectively) associated with a particular single sampling plan are based on $p_{1}, N, n$, and $A c$. The mathematical statisicics necessary for the calculation of Consumer's and Producer's Risks for the single sampling plans are discussed in Appendix B. However, a less detailed discussion is warranted at this point and is provided in the next two paragraphs.

In Section 3.2 the Consumer's Risk is defined as the probability of accepting a bad batch. In the context of this report, a "bad batch" is defined as an SDG with a fraction nonconforming $(p)$ greater than the maximum allowable fraction nonconforming $\left(p_{1}\right)$. The probability of accepting a bad batch is at a maximum for the first $p$ which is greater than $p_{1}$. The Consumer's Risks given in the fourth column of the tables in Appendix A correspond to this maximum.

The Froducer's Risk (probability of rejecting a good batch), provided in the fifth column of the tables in Appendix $A$, is not the maximum possible for the

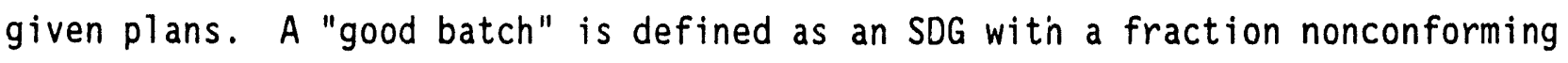
$(p)$ which is, at most, equal to the maximum allowable fraction nonconforming $\left(p_{1}\right)$. The maximum Producer's Risk occurs when $p$ is equal to $p_{1}$, but under this condition the maximum Producer's Risk is approximately equal to one minus the maximum Consumer's Risk. Since these two maximized risks sum to one, as one risk decreases the other risk must increase, and it is impossible to simultaneously control both of them. Producer's Risk can also be evaluated for any $p$ which is less than $p_{1}$. The Producer's Risks of Appendix A correspond to the probability of rejecting an SDG whose fraction nonconforming is approximately half of the maximum allowable fraction nonconforming, i.e., Producer's Risk is evaluated for $p \approx 0.5^{*} p_{1}$. The decision to evaluate Producer's Risk at one half of the maximum allowable fraction nonconforming is based on engineering judgement. Producer's Risk, evaluated this way for a particular sampling plan, provides the likelihood of rejecting an SDG whose number of nonconforming reported values is actually very acceptable.

The last column of the tables in Appendix A corresponds to the plan's sampling fraction ( $n / N$ expressed in percent). The sampling fraction gives some indication of the potential cost reduction associated with using the single 
sampling plan. It is important to note the sampling fraction reflects the potential cost reduction that one could expect over time, provided the contract laboratory is performing well relative to $p_{1}$ ( when $\left.p<<p_{1}\right)$. In other word, as the actual fraction nonconforming increases, the overall probability of rejection of the SDGs will increase, thus subsequent one-hundred percent inspection is more likely, thereby increasing the cost of inspection.

\subsection{The Assumptions Associated With the Single Sampling Plans}

All of the single sampling plans provided in Appendix A were generated under the following assumptions, which were established based on discussions with the SMO:

Given $p_{1}$ and $N$,

1) Consumer's and Producer's Risks, denoted by $\alpha$ and $\beta$ respectively, are equally important from the perspective of the project manager and should be at most $20 \%$, and

2) nonconformities occur randomly in the SDG

3) all analytes and/or samples are of equal importance to the project manager

The first assumption essentially says, find an acceptance sampling plan such that we will accept SDGs with a fraction nonconforming which is approximately half of $p_{1}$ about $80 \%$ of the time, and we will reject SDGs with a fraction nonconforming that is slightly more than $p_{1}$ about $80 \%$ of the time. An acceptance sampling plan meeting these requirements is called an 80-80 plan. All of the single sampling plans in Appendix $A$ are at least 80-80 plans.

The requirement of randomly occurring nonconformities is the second assumption. For the discussion of this assumption refer to Figures 1 through 4 . In viewing the figures consider an SDG containing four samples, each analyzed for 
four particular analytes. The reported values associated with this SDG can be thought of as elements of a matrix where the row of the matrix corresponds to the sample $\left(S_{i}\right)$ and the column of the matrix corresponds to the analyte $\left(A_{j}\right)$, as depicted in the figures. Figure 1 depicts a situation where the nonconforming reported values, denoted with and $x$, occur randomly.

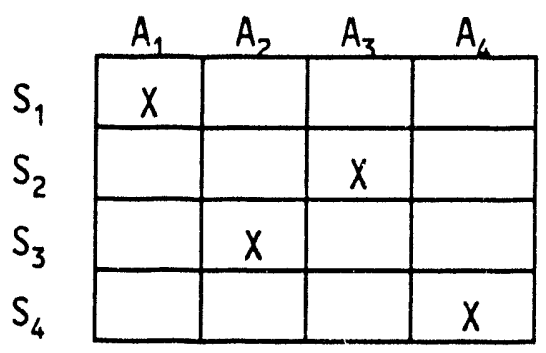

Figure 1. Randomly occurring nonconforming reported values.

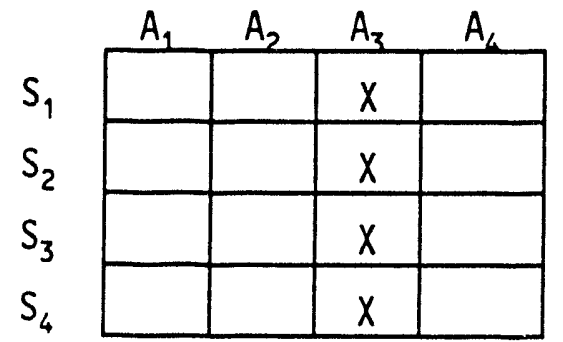

Figure 3. Nonconforming reported values correlated with analytes.

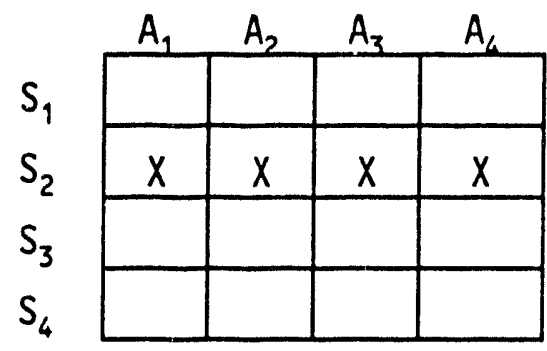

Figure 2. Nonconforming reported values correlated with samples.

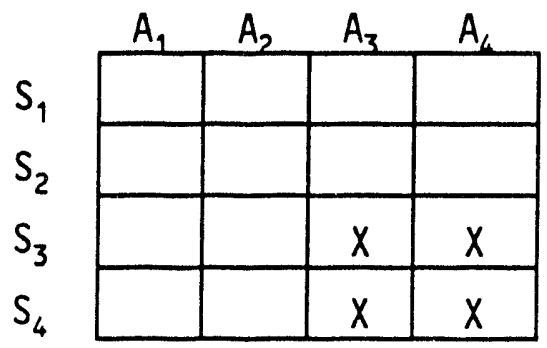

Figure 4. Nonconforming reported values correlated with samples and analytes.

Figure 2 depicts a different situation, one where the nonconforming reported values are correlated with the samples. The situation depicted in Figure 2 could arise, for example, if the \% solids for Sample 2 was computed incorrectly, thereby affecting all the reported values for that sample.

The situation depicted in Figure 3 is one where the nonconforming reported values are correlated with the analytes. In particular, all the Analyte 3 reported values are nonconforming. This situation could arise, for example, if the samples were analyzed for the wrong set of analytes. 
Finally, Figure 4 depicts a situation that is a combination of the previous two scenarios, i.e., the nonconforming reported values are correlated with both the analyte and the sample. Use of the wrong dilution factor could generate an SDG of this nature.

If patterns, such as those depicted in Figures 2 - 4, are present in an SDG, then the true fraction nonconforming will be estimated properly provided the statistical sample of size $n$ (reported values) is randomly selected from among al1 $\mathrm{N}$ sample/analyte combinations. If these sorts of patterns can be screened prior to SRV then alternate statistical sampling techniques could be employed. For instance, in performing the SRV it is most efficient for the validator to inspect reported values in a systematic way, i.e., inspecting all reported values for a particular sample before moving on to the next sample. If it is reasonable to assume that nonconforming reported values occur randomly in the SDG (no patterns), then the statistical sample size $n$ given in the tables of Appendix $A$ could be split into sets of 23 and the SRV inspection process could be performed by randomly selecting $n^{\prime}=n / 23$ samples and performing SRV on all the reported values for the $n^{\prime}$ samples.

In the examples depicted by the figures the true fraction nonconforming is $25 \%(4 / 16 \times 100 \%)$, regardless of which scenario exists. Suppose that the acceptance sampling $\mathrm{plan}$ for this SDG specified a maximum allowable fraction nonconforming of $20 \%\left(p_{1}=20 \%\right)$, with a statistical sample size of four $(n=4)$, and an acceptance criterion of zero $(A c=0)$. The proposed $n^{\prime}$ method described above would specify that one sample $\left(n^{\prime}=n / 4\right)$ be randomly selected to receive SRV on all of its reported values. Note that the $n^{\prime}$ method would catch the $25 \%$ fraction nonconforming in Figures 1 and 3 regardless of which sample was selected. But in the scenarios described in Figures 2 and 4 false acceptance may occur depending on the sample chosen for inspection. Thus, it is crucial that there be no patterns present in order to justify the $n^{\prime}$ method on an SDG. Methods for screening these patterns will be addressed in a subsequent Standard Operating Procedure (SOP). 
Lastly, the acceptance sampling plans contained in this report were generated under the assumption that all the reported values are equally important to the project manager (third assumption). In other words, no particular sample or analyte is of special importance in the SDG. Situations arise where this assumption may not be true. For example, a particular sample in the SDG may be of special interest to the project manager and subsequently they may want all the reported values for that sample to be processed through SRV. The acceptance sampling plans can sti?l be applied to the remainder of the SDG. These type of situations will be addressed in more detail in a subsequent SOP.

In viewing the tables in Appendix $A$, notice that for a given $p_{1}$, finding a single sampling plan which satisfies the first two assumptions becomes more difficult as $N$ decreases. In the cases where $N$ is small it was necessary to require $100 \%$ inspection since no sampling plans were found which met these two assumptions. However, this becomes less of a problem as $p_{1}$ increases.

For a given choice of $N$ and $p_{1}$ there may be many single sampling plans which satisfy the above assumptions. To construct the tables in Appendix $A, a$ computer program (written in the SAS ${ }^{\circledR}$ programming language, a commercial statistics software package), was used to generate a collection of single sampling plans satisfying the assumptions for each combination of $p_{1}$ and $N$. Each collection of plans was then trimmed down to one "best" $p l a n$ for that $p_{1}$ and $N$, based on the relative size of $\alpha$ and $\beta$.

The use of these tables is best illustrated through an example. If the project manager requires an error rate of no more than $10 \%$ in the reported values associated with the SDGs in a case, then Table 2, with $p_{1}=10 \%$, in Appendix $A$ would be used. Once $p_{1}$ has been chosen, the validator performs the SRV on all the SDGs within the project manager's case according to the corresponding table. Suppose a data package arrives containing 414 reported values (18 samples). Table 2 specifies that 115 of the reported values (5 samples) should be randomly selected from the data package for the SRV process. If eight or fewer reported values in the statistical sample are nonconforming, then the SDG is accepted as good with respect to SRV. If the number of nonconforming reported values in the statistical sample exceeds eight, then the SDG is rejected as bad with respect 
to SRV. When rejection occurs the validator will inspect all the remaining reported values in the data package. With this acceptance sampling plan ( $N=414$, $n=115, A c=8$ ) the likelihood of accepting an SDG with an error rate slightly worse than $10 \%$ is given by the Consumer's Risk of $12.3 \%$. Similarly, the likelihood of rejecting an SDG with an error rate that is approximately $5 \%$ (half of $10 \%$ ) is given by the Producer's Risk of $9.4 \%$. This acceptance sampling plan requires inspection of about $28 \%$ of the reported values, provided the contract laboratory's quality level is significantly better than the $10 \%$ error rate. 


\section{DOUBLE SAMPLING PLANS}

Double sampling may require that up to two statistical samples, one of size $n_{1}$ and a second of size $n_{2}$, be taken for the sake of determining the acceptability of a batch. There are two sets of acceptance/rejection criteria at each stage of the statistical sampling. The advantage of double sampling is that, on the average, the sampling fraction may be less than the single sampling plan while maintaining the same risk levels, provided the Producer's actual quality level is significantly better than the maximum allowable fraction nonconforming. The disadvantage of double sampling is that it is a more complicated procedure than single sampling. Thus, even though there may be improvement in either the sampling fraction or the risks, the cost of implementation may be more for double sampling.

Double sampling plans were investigated as an acceptance sampling approach to SRV. The conclusion of the investigation is that it would not be cost effective to implement. However, double sampling is discussed and the efforts of the investigation are documented in Appendix $C$. 


\section{RECOMMENDATIONS}

In implementing acceptance sampling to the SRV process the following recommendations are suggested.

1) Project managers and SMO personnel should be asked to review this document and the proposed single sampling plans for SRV with the following question in mind: Are the given levels of $p_{1}$ and Consumer's and Producer's Risks specified by these plans appropriate for their data validation needs? other single sampling plans can be constructed based on new criteria if necessary.

2) The SMO should develop a cost model which relates the cost of validation to the number of reported values inspected, and other factors, then assess whether the single sampling $\mathrm{plans}$ are cost effective. If they are, determine what sort of reduction in average statistical sample size is required in order justify generating double sampling plans.

3) Consideration should be given to whether it is appropriate to assign one of the particular single sampling plans provided in this report $\left(p_{1}=5 \%\right.$, $10 \%$, or $20 \%$ ) to each of the data validation levels $B$ and $C$. If not, determine the appropriate constraints for generating a single sampling plan for assignment to these data validation levels. 


\section{REFERENCES}

EG\&G Idaho, Environmental Restoration Department Requirements and Guidance for Data Validation, EGG-WM-10045, Rev 0, June 1992.

USEPA Contract Laboratory Program, Statement of Work for Inorganic Analysis, Multi-Media Multi-Concentration, Document Number ILMO2.0.

EG\&G Idaho, Standard Operating Procedure for Inorganic Data Validation, SMO-SOP12.1.5, September 1991.

EG\&G Idaho, Standard Operating Procedure for Levels of Method Validation, SMOSOP-12.1.1, Ju1y 1991 .

USEPA, Laboratory Data Validation Functional Guidel ines for Evaluating Inorganics Analyses, Compiled by Ruth Bleyler, July 1988.

Duncan, Acheson J., Quality Control and Industrial Statistics, Homewood IL: IRWIN, 1986. 


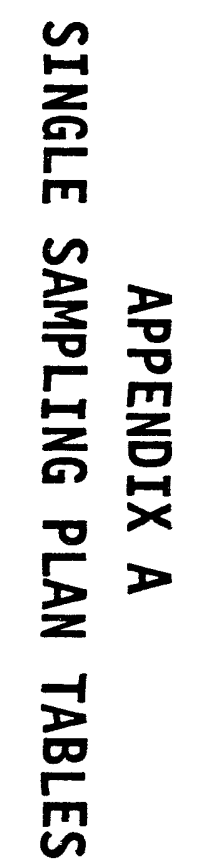


Table 1. Single sampling plan with $p_{1}=5 \%$. $^{a}$

\begin{tabular}{|c|c|c|c|c|c|}
\hline $\begin{array}{c}\text { Size of SDG } \\
(N) \\
\end{array}$ & $\begin{array}{c}\text { Statistical } \\
\text { Sample Size } \\
(n)\end{array}$ & $\begin{array}{c}\text { Acceptance } \\
\text { Number } \\
(A C) \\
\end{array}$ & $\begin{array}{c}\text { Consumer } \\
\text { Risk } \\
(\alpha \%) \\
\end{array}$ & $\begin{array}{c}\text { Producer } \\
\text { Riskc } \\
(\beta \%) \\
\end{array}$ & $\begin{array}{l}\text { Sampling } \\
\text { Fraction } \\
(n / N \%) \\
\end{array}$ \\
\hline $23-115$ & $N A^{d}$ & $N A$ & NA & $N A$ & 100 \\
\hline 138 & 92 & 3 & 16.8 & 0.0 & 67 \\
\hline 161 & 92 & 3 & 12.8 & 10.4 & 57 \\
\hline 184 & 115 & 4 & 12.1 & 9.2 & 63 \\
\hline 207 & 115 & 4 & 15.8 & 5.1 & 56 \\
\hline 230 & 115 & 4 & 18.7 & 10.6 & 50 \\
\hline 253 & 161 & 5 & 5.3 & 6.4 & 64 \\
\hline 276 & 161 & 5 & 7.0 & 13.5 & 58 \\
\hline 299 & 161 & 5 & 8.5 & 8.9 & 54 \\
\hline 322 & 161 & 6 & 16.0 & 3.3 & 50 \\
\hline 345 & 161 & 6 & 17.9 & 5.9 & 47 \\
\hline 368 & 161 & 6 & 19.6 & 4.0 & 44 \\
\hline 391 & 184 & 6 & 8.9 & 12.5 & 47 \\
\hline 414 & 184 & 6 & 9.9 & 9.3 & 44 \\
\hline 437 & 184 & 6 & 10.9 & 12.4 & 42 \\
\hline 460 & 184 & 6 & 9.2 & 15.5 & 40 \\
\hline
\end{tabular}

a. This $p l a n$ is based on the size of the SDG being validated, assumes a maximum allowable fraction nonconforming of 5\%, and controls Consumer's and Producer's Risks at less than $20 \%$.

b. The Consumer's Risk is evaluated for the smallest fraction nonconforming that is larger than $5 \%$.

c. The Producer's Risks is evaluated for a fraction nonconforming that is approximately $2.5 \%$.

d. $N A=$ Not Applicable due to $100 \%$ inspection. 
Table 2. Single sampling plan with $p_{1}=10 \%$. $^{a}$

\begin{tabular}{cccrrr}
\hline $\begin{array}{c}\text { Size of SDG } \\
(N)\end{array}$ & $\begin{array}{c}\text { Statistical } \\
\text { Sample Size } \\
(n)\end{array}$ & $\begin{array}{c}\text { Acceptance } \\
\text { Number } \\
(A C)\end{array}$ & $\begin{array}{c}\text { Consumer } \\
\text { Risk } \\
(\alpha \%)\end{array}$ & $\begin{array}{c}\text { Producer } \\
\text { Risk } \\
(\beta \%)\end{array}$ & $\begin{array}{c}\text { Sampling } \\
\text { Fraction } \\
(n / N \%)\end{array}$ \\
\hline $23-46$ & $N A^{d}$ & $N A$ & $N A$ & $N A$ & 100 \\
69 & 46 & 3 & 16.1 & 0.0 & 67 \\
92 & 69 & 5 & 6.6 & 0.0 & 75 \\
115 & 69 & 5 & 14.5 & 4.3 & 60 \\
138 & 69 & 5 & 19.9 & 5.8 & 50 \\
161 & 69 & 5 & 17.8 & 6.5 & 43 \\
184 & 92 & 6 & 7.2 & 8.4 & 50 \\
207 & 92 & 6 & 9.3 & 9.0 & 44 \\
230 & 92 & 6 & 8.4 & 15.2 & 40 \\
253 & 92 & 6 & 10.0 & 14.7 & 36 \\
276 & 92 & 6 & 11.3 & 14.3 & 33 \\
299 & 92 & 6 & 12.6 & 14.0 & 31 \\
322 & 92 & 6 & 11.4 & 13.8 & 29 \\
345 & 92 & 6 & 12.5 & 13.5 & 27 \\
368 & 92 & 6 & 13.4 & 13.3 & 25 \\
391 & 92 & 6 & 12.4 & 16.5 & 24 \\
414 & 115 & 8 & 12.3 & 9.4 & 28 \\
437 & 115 & 8 & 13.2 & 9.2 & 26 \\
460 & 115 & 8 & 12.2 & 9.1 & 25 \\
\hline
\end{tabular}

a. This plan is based on the size of the SDG being validated, assumes a maximum allowable fraction nonconforming of $10 \%$, and controls Consumer's and Producer's Risks at less than $20 \%$.

b. The Consumer's Risk is evaluated for the smallest fraction nonconforming that is larger than $10 \%$.

c. The Producer's Risks is evaluated for a fraction nonconforming that is approximately $5 \%$.

d. $N A=$ Not Applicable due to $100 \%$ inspection. 


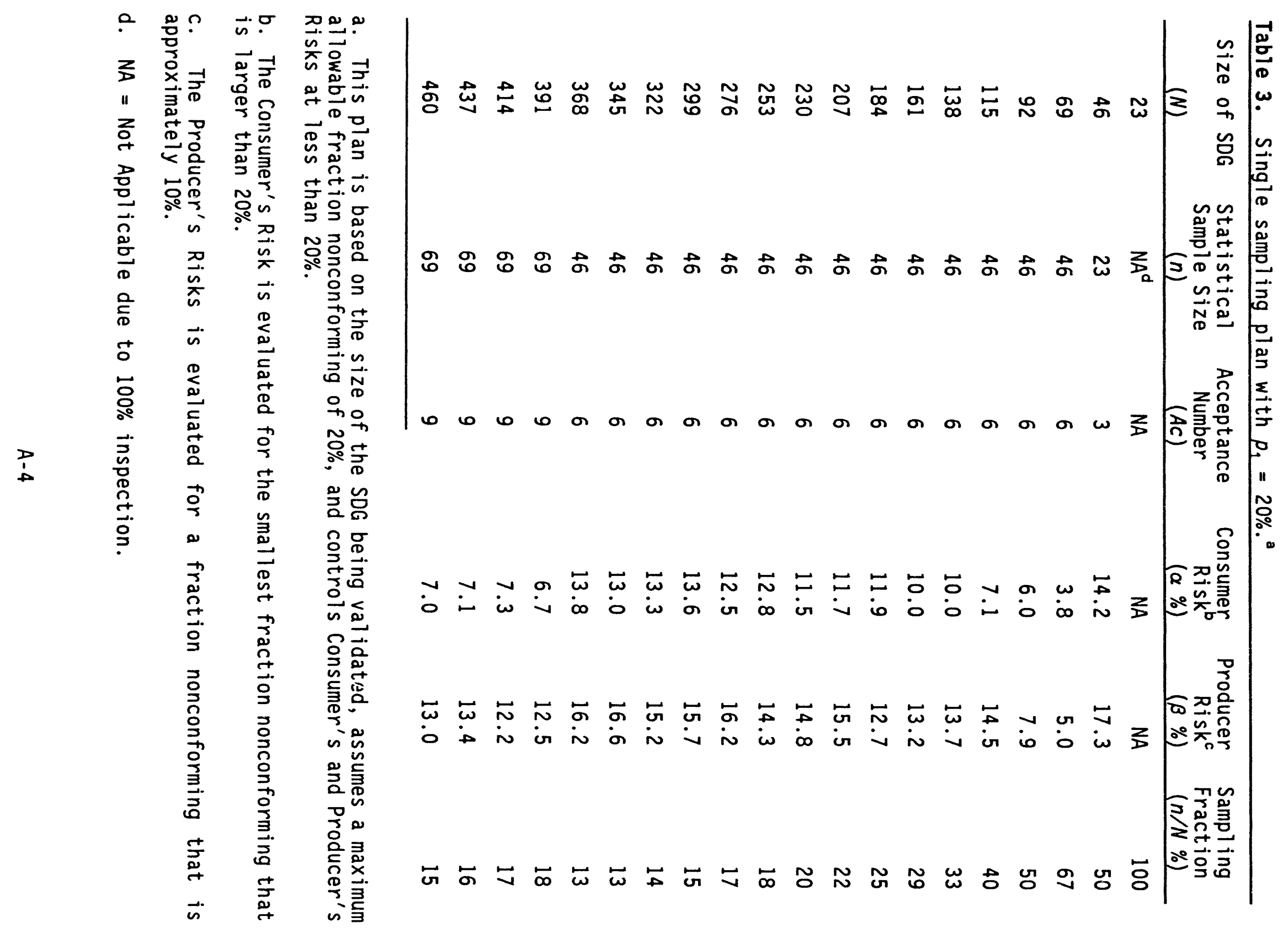




\section{APPENDIX B}

MATHEMATICAL STATISTICS OF THE SINGLE SAMPLING PLANS 


\section{B-1 NOTATION}

The following conventions in notation will be used throughout Appendix B:

$N=$ batch size, the number of units contained in the batch

$k$ = the actual number of nonconforming units contained in the batch

$p_{1}=$ the maximum allowable fraction nonconforming in the batch, specified as either a fraction or a percentage

$d_{1}=$ the maximum allowable number of nonconforming units in the batch, based on $p_{1}\left(d_{1}\right.$ is the largest integer such that $\left.d_{1} / N \leq p_{1}\right)$

$n$ = statistical sample size, the number of units to be randomly selected from the batch for inspection

$x=$ the number of nonconforming units in the statistical sample

$A c=$ maximum allowable number of nonconforming units in the statistical sample (if $X \leq A c$, then the batch is accepted; if $X>A c$, then the batch is rejected)

$\alpha=$ probability of falsely accepting a batch, also called Consumer's Risk

$=P$ (accept the batch given that it is "bad")

$=P\left(X \leq A C \mid k>d_{1}\right)$

$\beta=$ probability of falsely rejecting a batch, also called Producer's Risk

$=P$ (reject the batch given that it is "good")

$=P\left(X>A C \mid k \leq d_{1}\right)$

$p_{2}=$ a fraction nonconforming which is no greater than $p_{1}$, expressed as either a fraction or a percentage

$d_{2}=$ the number of nonconforming units most closely defined by $p_{2}$, i.e., $p_{2} \star N$ rounded to the nearest integer 


\section{B-2 THE HYPERGEOMETRIC PROBABILITY DISTRIBUTION}

Given a batch of size $N$ which contains $k$ nonconforming units and a statistical sample size of $n$, the number of nonconforming units in the statistical sample $(X)$ is distributed as a hypergeometric random variable. The probability that the statistical sample will contain exactly $r$ nonconforming units is denoted by $h(N, n, k, r)$ and is defined by the Hypergeometric Probability Distribution:

$P(X=r)=h(N, n, k, r)=\frac{C(k, r) C(N-k, n-r)}{C(N, n)}$ for $r=0,1,2, \ldots, k$

where $C(a, b)=\frac{a !}{(a-b) ! b !}$

and $a !$ denotes the product $a(a-1)(a-2) \ldots(2)(1)$.

The probability that the statistical sample will contain at most $r$ nonconforming units is denoted by $H(N, n, k, r)$ and is defined by the Cumulative Hypergeometric Probability Distribution:

$P(X \leq r)=H(N, n, k, r)=\sum_{i=0}^{r} P(X=i)=\sum_{i=0}^{r} \frac{C(k, i) C(N-k, n-i)}{C(N, n)}$ 


\section{B-3 DETERMINATION OF CONSUMER'S AND PRODUCER'S RISKS}

The determination of the Consumer's and Producer's Risks associated with a particular single sampling plan is based on $N, n, A c, p_{1}$, and $p_{2}$, as defined in the previous notation section.

\section{B-3.1 Determination of Consumer's Risk}

To determine the value of Consumer's Risk associated with a particular single sampling plan the Cumulative Hypergeometric Probability Distribution is employed. As defined in Section 3.2, Consumer's Risk is the probability of falsely accepting a batch based on the statistical sample results. When false acceptance occurs it means that a batch has been accepted when the fraction nonconforming is actually worse than what the Consumer is willing to tolerate.

The maximum allowable fraction nonconforming that the Consumer is willing to tolerate is denoted by $p_{1}$. Consumer's Risk is at a maximum when the number of nonconforming units in the batch is one more than the maximum allowable number of nonconforming units, i.e., for $k=d_{1}+1$.

The maximized Consumer's Risk, $\alpha$, is provided in the tables of Appendix A, and is calculated as follows:

$\alpha=P\left(X \leq A C\right.$, given $\left.k=d_{1}+1\right)$

$=H\left(N, n, d_{1}+1, A C\right)=\sum_{i=0}^{A C} \frac{C\left(d_{1}+1, i\right) C\left(N-\left(d_{1}+1\right), n-i\right)}{C(N, n)}$ 


\section{B-3.2 Determination of Producer's Risk}

The Cumulative Hypergeometric Probability Distribution is used in the calculation of the Producer's Risk associated with a single sampling plan. The Producer's Risk is the probability of falsely rejecting the batch based on the statistical sample results. When false rejection occurs it means that a batch had been rejected when the fraction nonconforming is actually no worse than what the Consumer is willing to tolerate.

The maximum allowable fraction nonconforming that the Consumer is willing to tolerate is denoted by $p_{1}$. Producer's Risk is maximized for the case in which the true number of nonconforming units in the batch is equal to the maximum allowable, or when $k=d_{1}$. But it is frequently more desirable to evaluate the Producer's Risk when the fraction nonconforming is much better than $p_{1}$, as this provides a perspective on the $\mathrm{plan}$ 's propensity to discriminate against good batches. The decision as to which value of fraction nonconforming is to be used in the evaluation of Producer's Risk is somewhat arbitrary. The Producer's Risks associated with the single sampling $\mathrm{plans}$ provided in this report are calculated based on a fraction nonconforming which is approximately half of the maximum allowable, or $p_{2} \approx 0.5 * p_{1}$. This is accomplished with $p_{2}=d_{2} / N$, where $d_{2}$ is obtained by rounding $0.5 * p_{1} * N$ to the nearest integer. Subsequently, Producer's Risk $(\beta)$ is calculated as follows:

$\beta=P\left(X>A C\right.$, given $\left.k=d_{2}\right)=1-P\left(X \leq A C\right.$, given $\left.k=d_{2}\right)$

$$
=1-H\left(N, n, d_{2}, A C\right)=1-\sum_{i=0}^{A C} \frac{C\left(d_{2}, i\right) C\left(N-\left(d_{2}\right), n-i\right)}{C(N, n)}
$$




\section{APPENDIX C \\ MATHEMATICAL STATISTICS OF THE DOUBLE SAMPLING PLANS}




\section{C-1 NOTATION}

The following conventions in notation will be used throughout Appendix C:

$N=$ batch size, the number of units contained in the batch

$k=$ the actual number of nonconforming units contained in the batch

$p_{1}=$ the maximum allowable fraction nonconforming in the batch, specified as either a fraction or a percentage

$d_{1}=$ the maximum allowable number of nonconforming units in the batch, based on $p_{1}\left(d_{1}\right.$ is the largest integer such that $\left.d_{1} / N \leq p_{1}\right)$

$n_{1}$ = statistical sample size for the first stage of acceptance sampling

$n_{2}=$ statistical sample size for the second stage of acceptance sampling

$X_{1}=$ the number of nonconforming units in the first statistical sample

$X_{2}=$ the number of nonconforming units in the second statistical sample

$A c_{1}=$ acceptance criterion for the first statistical sample (if $X_{1} \leq A c_{1}$, then the batch is accepted)

$R e_{1}=$ rejection criterion for the first statistical sample (if $X_{1} \geq R e_{1}$, then the batch is rejected)

$A c_{2}=$ acceptance criterion for the second statistical sample (if $\left(X_{1}+X_{2}\right) \leq A c_{2}$, then the batch is accepted, otherwise the batch is rejected)

$R e_{2}=$ rejection criterion for the second statistical sample

(if $\left(X_{1}+X_{2}\right) \geq R e_{2}$, then the batch is accepted, otherwise the batch is rejected) Note: $R_{2}=A c_{2}+1$

$\alpha=$ probability of falsely accepting a batch, also called Consumer's Risk $=P$ (accept the batch given that it is "bad")

$\beta=$ probability of falsely rejecting a batch, also called Producer's Risk

$=P$ (reject the batch given that it is "good")

$p_{2}=$ a fraction nonconforming which is no worse than $p_{1}$, expressed as either a fraction or a percentage

$d_{2}=$ the number of nonconforming units most closely defined by $p_{2}$, i.e., $p_{2} \star N$ rounded to the nearest integer 


\section{C-2 INTRODUCTION TO DOUBLE SAMPLING}

Double sampling may require that up to two statistical samples be taken for the sake of determining the acceptability of a batch. To determine the acceptability of a batch using a double sampling plan for attributes, a statistical sample is randomly drawn from the batch and inspected. If the number of nonconforming units in the first statistical sample $\left(X_{1}\right)$ is less than or equal to a predetermined number $\left(A c_{1}\right)$, the batch is accepted. If the number of nonconforming units in the first statistical sample is $R e_{1}$ or more, the batch is rejected and all of the reported values are subjected to SRV. If the number of nonconforming units in the first statistical sample is between $A c_{1}$ and $R e_{1}$, another statistical sample is taken of size $n_{2}$. Note, in double sampling the first stage acceptance number $\left(A c_{1}\right)$ and the first stage rejection number $\left(R e_{1}\right)$ must differ by more than one, i.e., $R e_{1} \geq A c_{1}+2$. If they did not, then the sampling could never progress to the second stage and therefore the plan would essentially be a single sampling plan.

If a second statistical sample is needed, the number of nonconforming units in the second statistical sample $\left(X_{2}\right)$ is combined with $X_{1}$ and the total is compared to a second (combined) acceptance criterion, $A c_{2}$. If the total number of nonconforming units in the two statistical samples exceeds $A c_{2}$ the batch is rejected, otherwise it is accepted. A double sampling plan is completely specified by $n_{1}, A c_{1}, R e_{1}, n_{2}$ and $A c_{2}$.

The advantage of double sampling is that, on the average, the sampling fraction may be less than the single sampling plan while maintaining the same risk levels. In double sampling the final statistical sample size drawn from a batch will be equal to $n_{1}$ or $n_{1}+n_{2}$, depending on the true quality level of the batch and the statistical sample obtained. An average statistical sample size can be computed based on the probabilities associated with accepting at the first stage and accepting at the second stage, given the actual quality level $p$. When the Producer has good quality level relative to $p_{1}$ the average statistical sample size will lean toward $n_{1}$, which is typically less than the statistical sample size required for single sampling. The disadvantage of double sampling is that 
it is a more complicated procedure than single sampling. Even though there may be improvement in either the sampling fraction or the risks, the cost of implementation may be more than for single sampling. 


\section{C-3 DOUBLE SAMPLING PLANS FOR SAMPLE RESULTS VERIFICATION}

In generating double sampling $\mathrm{plans}$ for SRV it was decided to keep the risks approximately the same as those of the corresponding single sampling plan and try to reduce the sampling fraction.

For a given choice of $N$ and $p_{1}$ there may be many double sampling $p l a n s$ which satisfy the 80-80 assumption that was used for the single sampling plans. The actual assumptions used for generating double sampling plans are more stringent in that the Consumer's and Producer's Risks should be at least as good as those afforded by the single sampling plan, and the average statistical sample size must be considerably less than the $n$ of the single sampling plan for it to be cost effective.

In examining double sampling $\mathrm{plans}$ for the SRV application, a computer program was written (again in the SAS ${ }^{\otimes}$ programming language) which, for a given $p_{1}$ and $N$, would generate a collection of double sampling plans. Each collection was then searched for a best $p l a n$ which would satisfy the stringent assumptions imposed by the single sampling plan. This process is very tedious and time consuming for the double sampling plans. For efficiency it was initially done under the 80-80 assumption, but not the multiple of 23 statistical sample size assumption. Additionally, during the early computer programming runs, it was assumed that cyanide was included in the metals target analyte analysis. The conditions that the computer program was run under were with $N=480$ with a $p_{1}$ of $20 \%$, since these are conditions under which a comparable double sampling $p l a n$ is most likely to be found. The double sampling $\mathrm{plan}$ that was generated under these conditions is provided below. 
For $N=460$ and $p_{1}=20 \%$, the single sampling $p l$ an given in Table 3 of Appendix $A$ is:

\begin{tabular}{llll}
$n$ & AC & $\begin{array}{l}\text { Consumer's } \\
\text { Risk }\end{array}$ & $\begin{array}{l}\text { Producer's } \\
\text { Risk }\end{array}$ \\
\hline 69 & 9 & $7.0 \%$ & $13.0 \%$
\end{tabular}

For $N=480$ and $p_{1}=20 \%$, the best double sampling $p l a n$ found through the above generation process is:

\begin{tabular}{llllllll}
$n_{1}$ & $A c_{1}$ & $R e_{1}$ & $n_{2}$ & $A c_{2}$ & $\begin{array}{l}\text { Consumer's } \\
\text { Risk }\end{array}$ & $\begin{array}{l}\text { Producer's } \\
\text { Risk }\end{array}$ & $\begin{array}{l}\text { Average } \\
n\end{array}$ \\
\hline 34 & 3 & 7 & 33 & 9 & 11.5 & 11.4 & 47
\end{tabular}

In comparing the above single and double sampling plans, attention must be given to whether the reduction in statistical sample size from 69 (single sampling) to an average statistical sample size of 47 (double sampling) significantly overrides the increase in the cost of implementation. This may be difficult to assess without information regarding the ease of use of the single sampling plans. It is recommended that no more be done with the generation of double sampling plans until this information can be provided. Intuitively it seems that very little is gained through the use of double sampling plans for this application.

The calculations of the Consumer's and Producer's Risks associated with a particular double sampling plan are based on $p_{1}, N, n_{1}, A c_{1}, R e_{1}, n_{2}$, and $A c_{2}$. The mathematical statistics necessary for these calculations are discussed next. 


\section{C-4 DETERMINATION OF CONSUMER'S AND PRODUCER'S RISKS}

The determination of the Consumer's and Producer's Risks associated with a particular double sampling $\mathrm{plan}$ is more complicated than for single sampling $p l a n s$ because there are more parameters to the $p l a n$. For double sampling plans Consumer's and Producer's Risks are based on $N, n_{1}, n_{2}, A c_{1}, R e_{1}, A c_{2}, p_{1}$, and $p_{2}$, as defined in the previous notation section.

Using the functional notation defined for the Hypergeometric Probability Distribution and the Cumulative Hypergeometric Probability Distribution defined in Appendix B-2, the overall probability of rejecting, $P$ (reject), is derived as follows:

Let $R E J E C T 1=$ reject at first stage

CONTIN1 = continue at first stage

REJECT2 $=$ reject at second stage

Then

$P(R E J E C T 1)=P\left(X_{1} 2 R e_{1}\right.$, given $\left.N, n_{1}, k\right)$

$=1-P\left(X_{1} \leq\left(R e_{1}-1\right)\right.$, given $\left.N, n_{1}, k\right)$

$=1-H\left(N, n_{1}, k, R e_{1}-1\right)$

and

$P($ REJECT2 $)=P($ CONTIN1 and REJECT2)

$=P$ (CONTIN1) P(REJECT2, given CONTIN1). 
There are $R e_{1}-A c_{1}-1$ ways to continue at the first stage, i.e., $X_{1}=A c_{1}+1, A c_{1}+2, \ldots$, or $R e_{1}-1$. Therefore $P$ (REJECT2) becomes:

$P(R E J E C T 2)=\sum_{i=A c_{1}+1}^{R e_{1}-1} P\left(X_{1}=i\right.$, given $\left.N, n_{1}, k\right) P\left(X_{2} \geq R e_{2}-i\right.$, given $\left.N-n_{1}, n_{2}, k-i\right)$

$=\sum_{i=\lambda C_{1}+1}^{R e_{1}-1} P\left(X_{1}=i\right.$, given $\left.N, n_{1}, k\right)\left[1-P\left(X_{2} \leq R e_{2}-i-1\right.\right.$, given $\left.\left.N-n_{1}, n_{2}, k-i\right)\right]$

$=\sum_{i=\lambda c_{1}+1}^{R e_{2}-1} h\left(N, n_{1}, k, i\right)\left[1-H\left(N-n_{1}, n_{2}, k-i, R e_{2}-i-1\right)\right]$

And finally, the overall probability of rejection, given $N, n_{1}, n_{2}$, and $k$, is derived by combining Eq C-4.1 and Eq C-4.2:

$P($ reject $)=P($ REJECT1 $)+P($ REJECT2 $)$

$=\left\{\left[1-H\left(N, n_{1}, k, R e_{1}-1\right)\right]+\right.$

(Eq $C-4.3$ )

$\left.\sum_{i=A c_{1}+1}^{R \theta_{1}-1} h\left(N, n_{1}, k, i\right)\left[1-H\left(N-n_{1}, n_{2}, k-i, R \epsilon_{i}-i-1\right)\right]\right\}$

\section{C-4.1 Determination of Consumer's Risk}

Consumer's Risk is determined by evaluating Eq $C-4.3$ for $k=d_{1}+1$, given $N$, $n_{1}, n_{2}, A c_{1}, R e_{1}$, and $A c_{2}$, and subtracting the result from one (since 1 - $P($ reject $)=P($ accept $))$. 


\section{C-4.2 Determination of Producer's Risk}

Producer's Risk is determined directly by evaluating Eq $C-4.3$ for $k=d_{2}$, given $N, n_{1}, n_{2}, A c_{1}, R e_{1}$, and $A c_{2}$. For this report $d_{2}$ was calculated as $0.5 * p_{1} * N$ rounded to the nearest integer, thereby making $p_{2}=d_{2} / N$ approximately half of $p_{1}$. 


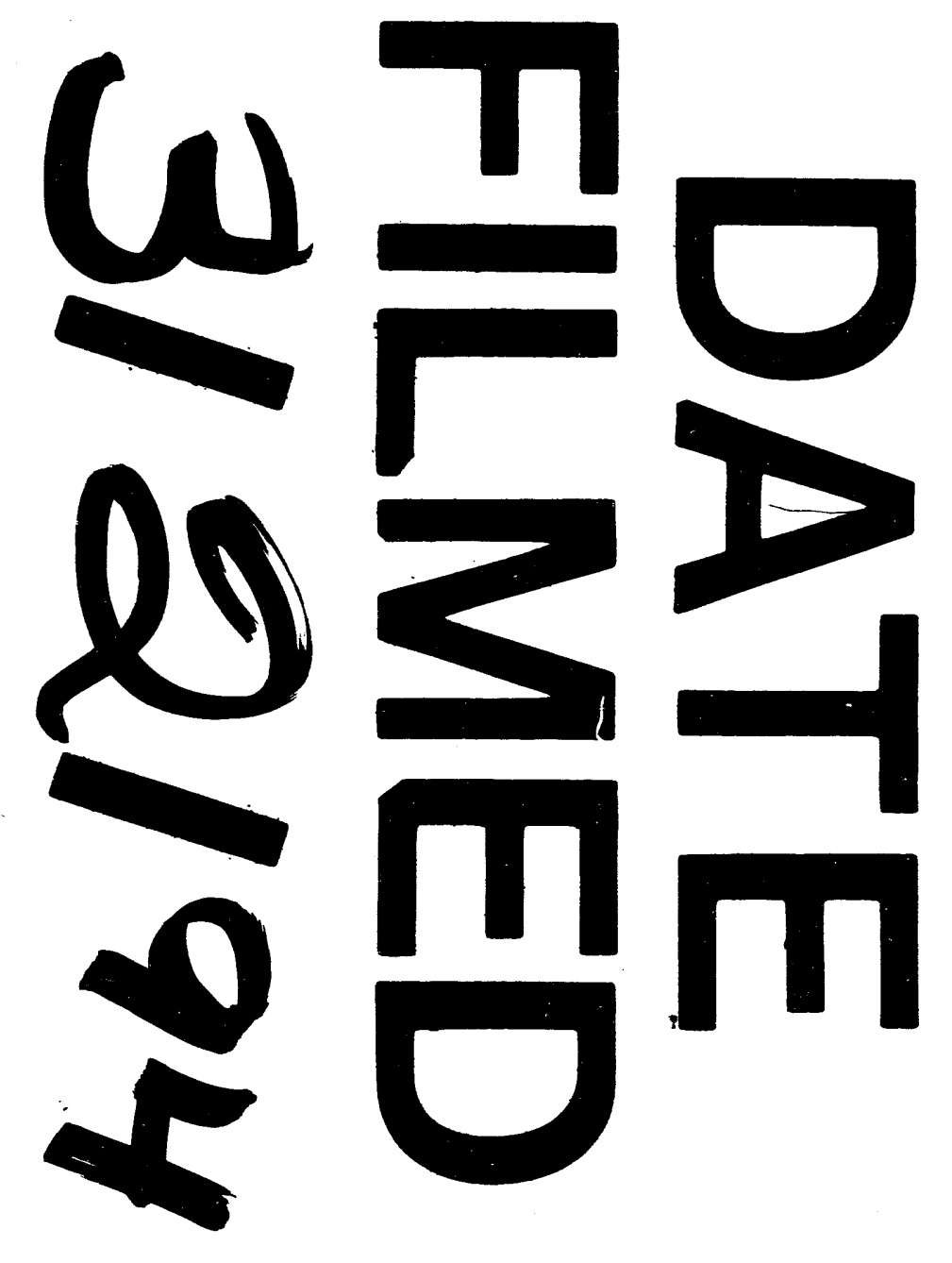


